\title{
White sponge naevus and ocular coloboma
}

\author{
S Wright, I S Levy
}

\begin{abstract}
White sponge naevus occurred in association with coloboma of the iris in six members of one family, across three generations. The association of these two dominantly inherited conditions has not to our knowledge previously been described.
\end{abstract}

There are several previous reports of an association between coloboma of the eye and other developmental abnormalities. ${ }^{1-4}$ Colobomas are usually inherited as an autosomal dominant characteristic.

White sponge naevus was first described by Cannon in 1935,5 but has a number of other names including hereditary leucokeratosis ${ }^{6}$ and white folded gingivostomatitis. ${ }^{7}$ The condition is also inherited as an autosomal dominant characteristic, but has not to our knowledge been described in association with ocular coloboma.

We report a family pedigree in which there is an association between white sponge naevus and iris coloboma; it confirms previous reports that both these congenital abnormalities are inherited in an autosomal dominant manner.

The Royal Free Hospita

School of Medicine,

Pond Street,

London NW3 2QG

S Wright

Department of

Ophthalmology

The Royal London

Hospital,

London E1 1BB

I S Levy

Correspondence to:

Dr Wright.

Accepted 18 November 1990

\section{CASE 1}

A boy aged 3 years (IV.6 in fig 1) was referred to the department of dermatology at this hospital because of sparse hair. When seen in the clinic, his hair was noted to be sparse, but the discrete areas of hair loss described by his mother were not seen. He had bilateral inferomedial colobo- mas of the iris (fig 2). Examination of his mouth showed a creamy white folded hyperkeratosis particularly affecting the buccal mucosa, but also the tongue, and pharynx. He was below the 3 rd centile for height and below the 10th centile for weight, but a full developmental assessment suggested that this was a constitutional short stature. Microscopy of his hair was normal. Slit lamp examination of his eyes and indirect ophthalmoscopy showed a normal retina and lens. Culture of mouth swabs failed to grow Candida spp and radiographic assessment showed that his bone age was consistent with his chronological age. At the age of $2 \cdot 1$ years he had a strangulated left indirect inguinal hernia repaired.

CASE 2

The mother of case 1 (III.4 in fig 1), who was aged 21 years, was noted to have bilateral iris colobomas at birth. During school dental examinations she had a folded, creamy white hyperkeratosis of the buccal mucosa (fig 3), which on histological examination was identical

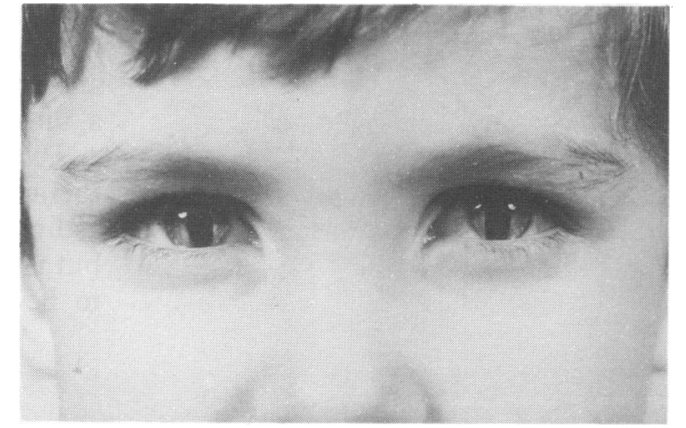

Figure 2 Eyes of case 1 showing bilateral inferomedial coloboma of the iris.

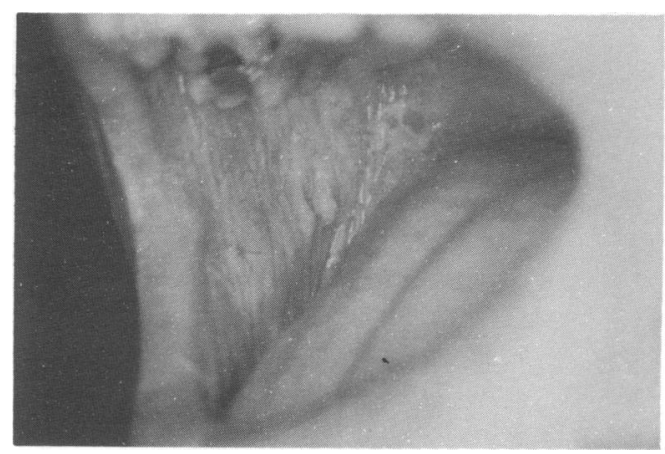

Figure 3 A creamy white folded hyperkeratosis of the buccal mucosa is extending back to the pharynx (case 2).
Figure 1 Family pedigree showing affected family members across four generations. Those affected family members examined by one of the authors $(S W)$ are indicated by an asterisk. The proband is indicated by an arrow.

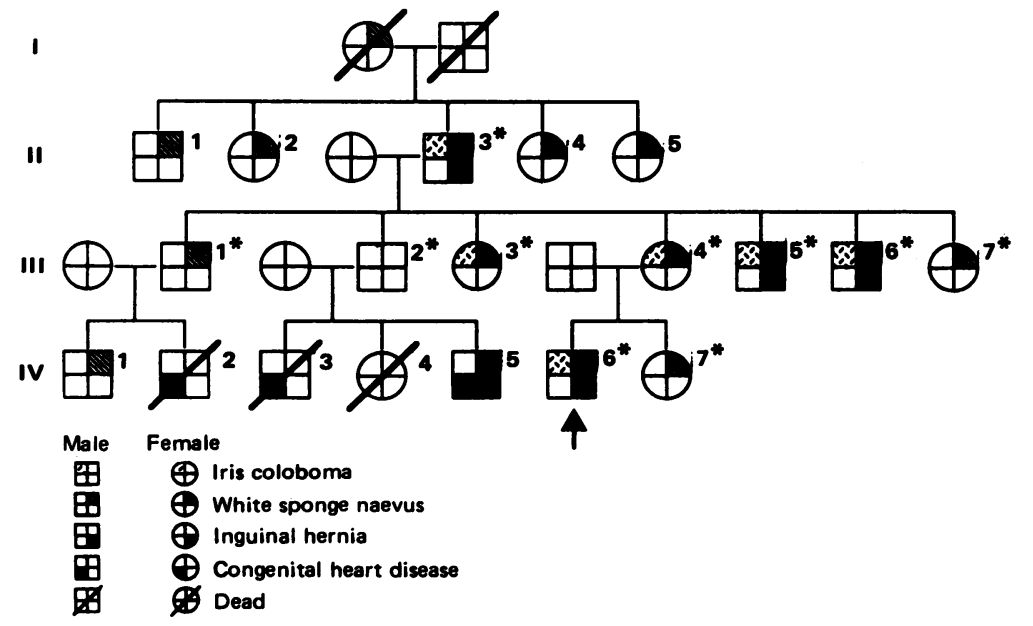


to that of her son. Despite persistently negative swabs, she was treated on several occasions with anticandida preparations, but with no success. She had no oral symptoms. Vaginal and rectal mucosa were unaffected.

Slit lamp examination and indirect ophthalmoscopy with a dilated iris showed a normal lens and retina. A biopsy specimen of the oral mucosa showed an acanthotic epithelium with intracelluar oedema and some vacuolation in the outer half. Maturation and stratification were normal, and there was no dyskeratosis. There was a light superficial dermal lymphocyte infiltrate (fig 4).

A family history was obtained from case 2, who was aware that her paternal grandmother had the same oral abnormality. The ocular abnormalities were present in her father's right eye. He was also examined and found to have coloboma limited to the iris and oral findings identical to those of his daughter. Subsequently a number of visits to various members of the family have enabled the construction of a reasonably complete family tree (fig 1 ).

In generation II, all five siblings have oral lesions and one out of five has ocular coloboma. In generation III, six out of seven siblings have oral lesions and four out of seven have iris coloboma. Every member of the family with iris coloboma also has white sponge naevus, although some members with white sponge naevus do not have iris coloboma. In the seven children so far born in generation IV, four have white sponge naevus and one of these four (the index case) also has bilateral iris colobomas. Three children in generation IV have died in infancy, one of agenesis of the left heart, one

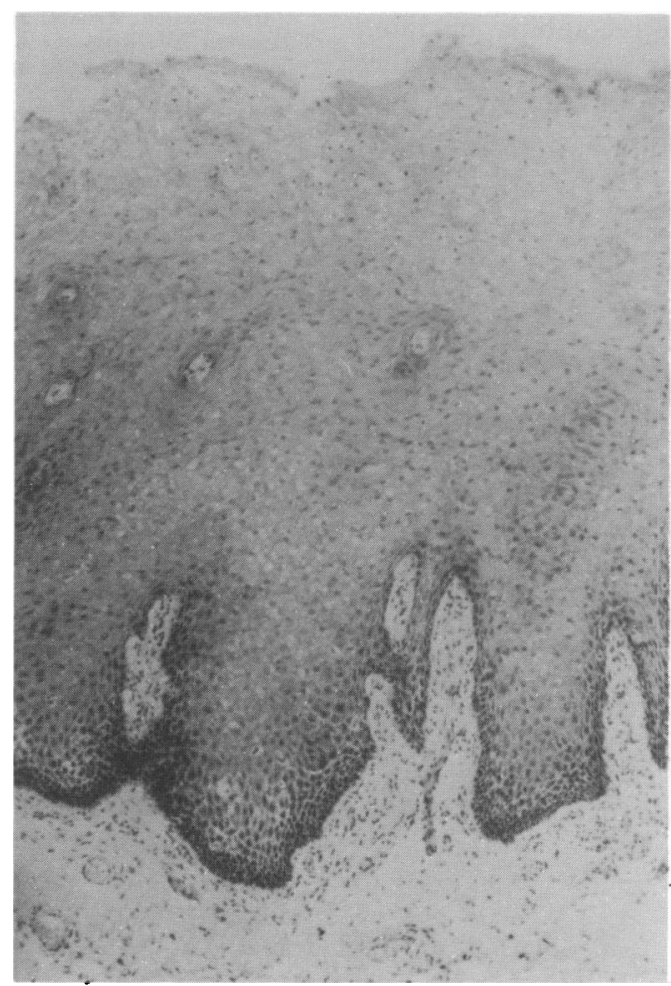

Figure 4 Biopsy specimen of the oral mucosa from case 2 shows acanthosis, with intracellular oedema affecting the epithelium and some vacuolation in the outer half. There is a light superficial dermal lymphocytic infiltrate.

(Hacmatoxylin and cosin, magnification 150.) with Fallot's tetralogy who died of infection after a Blalock shunt, and the other one of unknown cause during the first year of life. One surviving child has a small ventricular septal defect. Of the eight men with white sponge naevus in the whole family pedigree, five have had inguinal hernias reduced surgically during early childhood. Nothing remarkable was noted about these hernias at the time of operation. High resolution karyotyping of two adult members of the family is normal.

One of the authors (SW) has personally examined 10 affected family members (fig 1). No family member has been listed as affected unless they have either been seen by the author or have had the signs confirmed by at least two members of the family. Those family members who have not been examined are either out of the country or have refused to consent to physical examination.

\section{Discussion}

The differential diagnosis of white sponge naevus includes pachyonychia congenita, dyskeratosis congenita, Darier's disease, lichen planus, oral candidiasis, and hereditary benign intraepithelial dyskeratosis. White sponge naevus is usually congenital but may appear at any stage of infancy or childhood, and occasionally in adolescence. The oral lesions are typically widespread, affecting the palate, gingiva, floor of the mouth, and tongue as well as the buccal mucosa and comprise a thickened, folded, or corrugated epithelium with a spongy texture and a white opalescent hue. The condition is asymptomatic. This clinical picture alone is sufficient to make a positive diagnosis, and the histological features confirm it. The appearances described in fig 4 are typical, with, in particular, no dyskeratosis. There is acanthosis and usually more parakeratosis than is seen in this case. Oedema throughout the spongy layer is associated with vacuolation of keratinocytes. There may be a sparse lymphocytic infiltrate in the underlying dermis.

Witkop et al have reported an extended family pedigree in which 75 members of a family of $\mathbf{3 4 5}$ from an isolated rural community were affected by similar oral signs. ${ }^{8}$ Of those affected, $97 \%$ also had an ocular abnormality comprising a firm, raised wedge of tissue affecting the conjunctiva in the nasal and temporal perilimbal area. Histological examination of the oral mucosa in this congenital syndrome shows that it is distinguished from white sponge naevus by the prominent dyskeratosis, and the condition has been called 'hereditary benign intraepithelial dyskeratosis'.

Involvement of the rectal or vaginal mucosa, or both, has been reported in white sponge naevus, ${ }^{9}$ although the irregularity with which this is reported makes it unclear whether the involvement of mucosa other than that in the mouth represents variable expressivity of one genodermatosis, or represents a different genetic entity. ${ }^{10}$ Rectal or vaginal mucosal involvement is not a feature of this family.

Colobomas of the eyes result from the failure of the optic vesicle to close during the seventh 
or eighth week of fetal life, and may therefore affect any or all of the neuroectodermal structures derived from the optic vesicle-the iris, the ciliary body, choroid, optic disc, or optic nerve. They have been described in association with a variety of neuroectodermal syndromes, perhaps the best known of which is the Biemond syndrome II, comprising pituitary dwarfism associated with mental retardation, obesity, hypogonadism, postaxial polydactyly, and colobomas. Recently attention has been focused on the CHARGE association of colobomas, heart disease, atresia choanae, retarded growth, genital hypoplasia, and ear abnormalities. Iris colobomas have not to our knowledge been described in association with white sponge naevus, but the extent of the association in the family reported here serves to establish it as a real association.

The coexistence of white sponge naevus and iris coloboma in this family and the fact that all family members with either unilateral or bilateral iris coloboma also have white sponge naevus suggest that it is a single gene autosomal dominant disorder. If this were the case white sponge naevus would be expressed in all gene carriers, whereas iris coloboma would show variable expression.

Alternatively there could be two linked genes which have become separated by recombination in those family members who have white sponge naevus but not iris coloboma, but remain linked in those family members who show both characteristics. This seems less likely for several reasons. If iris coloboma resulted from a single dominant gene, one would expect it to be an isolated finding in some family members. As 10 of 19 family members have white sponge naevus without iris coloboma, the recombination rate of the proposed two genes would be roughly $50 \%$; this is not consistent with the genes being in close linkage.
None the less it is of interest that iris coloboma has not been described in the same patient as white sponge naevus before this family. The condition is visible, and white sponge naevus is unlikely to be missed at regular school dental examinations.

It is intriguing that the ocular coloboma in all the affected members of this family affects the iris only, as any part of the eye derived from the optic vesicle may theoretically be affected. This implies that the embryological development of the iris may be genetically separate from that of the rest of the chorioretinal tract.

The fact that three boys in generation IV have congenital heart disease and that inguinal hernia has occurred in infancy in at least five out of eight male infants suggest that a search among families with white sponge naevus or iris coloboma, or both, should be undertaken to find out if this apparent association is more than coincidence.

We thank Ms Wendy Straughair for typing the manuscipt.

1 Angelman H. Syndrome of coloboma with multiple congenital anomalies in infancy. $B M Y$ F $1961 ; i: 1212-4$.

2 Ho CK, Kaufman RL, Podos SM. Ocular colobomata, cardiac defect and other anomalies: a study of seven cases including two siblings. 7 Med Genet 1975;12:289-93.

3 Hittner HM, Hirsch NJ, Kreh GM, et al. Colobomatous microphthalamia, heart disease, hearing loss and mental retardation: a syndrome. $f$ Pediatr Ophthalmol Strabismus 1979;16:122-8.

4 Pagon RA, Graham JM, Zonana J, et al. Coloboma, congenital heart disease and choanal atresia with multiple abnortal heart disease and choanal atresia with multiple abnor5 malities: CHARGE association. F Pediat 1981;99:223-7. Dermatology and Syphilology (Chicago) 1935;31:365-71.

Dermatology and Syphilology (Chicago) 1935;31:365-71.
Haye KR, Whitehead FIH. Hereditary leucokeratosis of the Haye KR, Whitehead FIH. Hereditary leucokeratosis of
mucous membranes. Br f Dermatol 1968;80:529-33. 7 Everett FG, Noyes HJ. White folded gingivo-stomatitis. f Periodontol 1953;24:32-5.

8 Witkop CJ, Shankle CH, Graham JB, Murray MR Rucknagel DL, Byerly BH. Hereditary benign intraepithelial dyskeratosis: II Oral manifestations and hereditary transmission. Archives of Pathology 1960;70:696-711.

9 Zegarelli EV, Kutscher AH. Familial white folded hypertrophy of the mucous membranes. Oral Surgery 1957;10: 262-6.

10 Scott CR. Hereditary leucokeratosis, white mouth. $\mathcal{f}$ Pediatr 1966;68:768-71. 